\title{
Investigation and Study of Mode Splitting in Near Field Inductive Communication Systems
}

\author{
Hoa Doan Thanh and Johnson I. Agbinya
}

\begin{abstract}
Frequency splitting is a near field inductive communication phenomenon where the resonant frequency divides into many separate frequencies or to different modes. In this paper, we show that this phenomenon depends on the coupling coefficients or the natural response of the circuit by using the circuit theory to derive these splitting frequencies. Also, the rules for the general matrix that is used to solve for splitting frequencies are also demonstrated clearly. Mode splitting is observed for peer-to-peer, three coils and four coil systems due to the existence of the nearest and second neighbour interactions. In particular, two, three and four modes have been analysed for two, three, and four coil systems respectively. However, the number of modes for these systems can be changed according to the degree of coupling. The differences in the resultant splitting frequencies with and without the second neighbour interaction are shown in the simulation results. Furthermore, we assess the system performances regarding to power efficiency through the inductive transfer functions. Besides, either coupling coefficients at resonance or the simplified transfer functions in some specific scenarios can be obtained by having an insight into these transfer functions. Finally, we recognise and propose that splitting frequency phenomenon can be deployed to transmit signals at many frequencies concurrently.
\end{abstract}

Keywords-splitting frequencies, modes, the nearest and second neighbour interactions, coupling coefficients, conventional resonant frequency, power efficiency, transfer function

\section{INTRODUCTION}

D ATA and power transfer using magnetic flux coupling in near field communication has captured a lot of attention from researchers and telecommunication companies because it has a large number of applications in near field inductive communication devices, biomedical embedded devices, personal area networks, and wireless power transfer and data transmission. The name "near field inductive communication" stands for the very short distance between transceivers. In other words, most applications of inductive transceivers are based on the receivers within the flux bubble of the transmitter in order to induce current in the repeaters and then finally to enable the induced voltage in the receiver load. In general, the target of research work is to induce the maximum voltage in the receiver load to achieve the most efficient transmission by making both transmitter and receiver to resonate at the same frequency. This is the reason why transceivers are called resonators. The resonant frequency of LC circuits is well known as a function of inductance and capacitance of these components. The coupling coefficient however affects the resonant frequency. Therefore, the transceivers may perform in three

H. D. Thanh and J. I. Agbinya are with Electronic Engineering, La Trobe University, Melbourne, Australia (e-mails: hdoanthanh@students.latrobe.edu.au; J.Agbinya@latrobe.edu.au). operational regimes namely under coupling, critically coupling at the traditional resonant frequency and over coupling or strongly coupling. These regimes will result in different system performances and in terms of power, determine the efficiency of wireless power transfer in inductive systems as mentioned in other papers [1]-[4]. These papers recognised that systems perform best at either the critically or over coupling states for the purpose of power transfer efficiency.

The degree of coupling between transmitter, repeaters and receiver causes the transfer function or the scattering parameter (the ratio of the induced voltage at the load to the source voltage) to have two, three, four or $M$ modes according to the number of separate coils in the system. When the resonant frequency splits into several frequencies, the phenomenon is called frequency splitting. Hence if the transferred energy measurements are focused on the resonant frequency in such cases, significant system performance reduction will be observed. Mode splitting has been reviewed for peer-to-peer, three coil and four coil systems by a means of the coupled mode or circuit theory [1]-[7]. In a two coil system, two splitting frequencies have been shown in [5] without showing how to obtain those frequencies. Nevertheless, in [6] the author has shown clearly steps to obtain these two splitting frequencies by using circuit theory. In a three coil system, three modes have been analysed using the coupled mode theory [4]. In four coil systems, only two modes have been observed using the circuit theory [1]-[3]. However, papers [1] and [2] have demonstrated the relationships of the S-scattering parameter in terms of coupling coefficients and quality factors. Mode splitting behaviour of one system is not a result of the method of system analysis but is due to the natural response of system in certain circumstances [6], [7]. In [7], the authors have shown how to derive the splitting frequencies for three systems through the circuit theory analysis. In particular, two, three and four modes have been demonstrated for two, three, and four coil systems respectively. Therefore mode splitting is not a new idea [1]-[7]. However none of these papers paid attention to the influence of second neighbour mutual coupling interaction between repeaters and transmitter which in fact can affect the splitting frequencies. Besides, [6] and [7] have not shown how to obtain the general matrix and the Eigen values that result in the splitting frequencies. This paper eliminates the drawbacks of earlier works and also demonstrates more accurate values of the split frequencies obtained by considering the second neighbour interaction, albeit with more computation required.

By acknowledging mode splitting phenomenon, we can further analyse the system performances in the concern of power transfer. The voltage gains or the inductive transfer 
functions have been written in this paper to quantity the power efficiency at different frequencies. Moreover, the coupling coefficients at resonance can be found at resonance. Along with that, the simplified versions of transfer functions at resonance can be achieved for loosely coupled and low quality factor conditions. These results are somehow similar with paper [8] if the second neighbour interaction is excluded from system analysis. Otherwise, the differences again have been shown in this work.

Therefore, there are four main parts mentioned in the body graph. The first part is the circuit analysis for peer-to-peer, three coil and four coil systems where the splitting frequency derivation method is shown in order to confirm the reason for the resulting in mode splitting. After that, the rules for the general matrix used to solve those frequencies have been presented. The second part is simulations for splitting and resonant frequencies where the numbers of modes have been observed for each system with and without the second neighbour mutual coupling interaction. It is stated that the nearest and second neighbour coupling coefficients decide the number of modes in each system. For instance, four coil systems can have two, three or four modes in accordance with the degree of coupling. Moreover, it is investigated that the results for splitting frequencies are different for systems affected and unaffected by the second neighbour interaction. The next part mentions about transfer functions for inductive systems. In this section, all the voltage gains are derived for two, three and four coil systems in terms of angular frequencies. In addition, the voltage gains also are represented in terms of coupling coefficients and quality factors as to figure out the coupling coefficients at resonance and simplify the transfer functions at special conditions. The last section is about the simulations for voltages at different frequencies for power transfer efficiency purpose. After all, the conclusion provides the summary of achievements and future applications. In Appendix we give definitions for all variables used in the paper.

\section{CiRCUit ANALYSis}

In the following analyses we used the circuit theory Kirchhoff Voltage Law (KVL) to analyse circuits for all of the systems. Circuit theory formulation is more tractable since it presents less complex system consideration and computation.

\section{A. Two Coil System}

By following the method of analysis in [6], [7], the circuit can be analysed as the given expression:

$$
\left[\begin{array}{cc}
1 & k_{12} \sqrt{\frac{L_{2}}{L_{1}}} \\
k_{21} \sqrt{\frac{L_{1}}{L_{2}}} & 1
\end{array}\right]\left[\begin{array}{c}
I_{1} \\
I_{2}
\end{array}\right]=\frac{\omega_{0}^{2}}{\omega^{2}}\left[\begin{array}{c}
I_{1} \\
I_{2}
\end{array}\right]
$$

Equation (1) is equivalent to:

$$
\mathbf{A} \mathbf{v}=\lambda \mathbf{v}
$$

Where $\mathbf{A}$ is a $2 \times 2$ matrix, $\mathbf{v}$ is Eigen vector and $\lambda$ is Eigen value. Since $\operatorname{det}(\mathbf{A}-\lambda \mathbf{I})=0$, this leads to the Eigen value equation:

$$
(1-\lambda)^{2}=k_{12}^{2}
$$

It is noticed that two coil systems are simple and do not have the second neighbour interaction. Hence, Equation (3) results in two separate frequencies that are not related to the second coupling interaction same as in [5]-[7] as given below:

$$
\omega_{1}=\frac{\omega_{0}}{\sqrt{1+k_{12}}} ; \quad \omega_{2}=\frac{\omega_{0}}{\sqrt{1-k_{12}}}
$$

\section{B. Three Coil System}

In the three coil system, the second neighbour coupling has occurred. Thus, the same method in [7] is re-applied for natural response of this system with the effects of both the nearest and the second mutual coupling in order to achieve the result:

$$
\left[\begin{array}{ccc}
1 & k_{12} \sqrt{\frac{L_{2}}{L_{1}}} & -k_{13} \sqrt{\frac{L_{3}}{L_{1}}} \\
k_{12} \sqrt{\frac{L_{1}}{L_{2}}} & 1 & -k_{23} \sqrt{\frac{L_{3}}{L_{2}}} \\
-k_{13} \sqrt{\frac{L_{1}}{L_{3}}} & -k_{23} \sqrt{\frac{L_{2}}{L_{3}}} & 1
\end{array}\right]\left[\begin{array}{c}
I_{1} \\
I_{2} \\
I_{3}
\end{array}\right]=\frac{\omega_{0}^{2}}{\omega^{2}}\left[\begin{array}{c}
I_{1} \\
I_{2} \\
I_{3}
\end{array}\right]
$$

For the $3 \times 3$ matrix $\mathbf{A}$, the Eigen value equation is:

$$
(1-\lambda)^{3}-(1-\lambda)\left(k_{12}^{2}+k_{23}^{2}+k_{13}^{2}\right)+2 k_{12} k_{23} k_{13}=0
$$

The solutions to Equation (6) require numerical methods such as the fixed point or Newton-Raphson iteration. From Equation (6), it is learnt that the Eigen value is different from unity, which means that the splitting frequencies do not coincide with the conventional resonant frequency. However, this result changes if the second neighbour interaction is excluded from consideration in the three coil inductive circuit. In that case, Equation (6) turns into the following:

$$
(1-\lambda)^{3}-(1-\lambda)\left(k_{12}^{2}+k_{23}^{2}\right)=0
$$

Equation (7) gives the same result as in Dukju Ahn and Songcheol paper [7] as:

$$
\begin{gathered}
\omega_{1}=\frac{\omega_{0}}{\sqrt{1+\sqrt{k_{12}^{2}+k_{23}^{2}}}} ; \\
\omega_{2}=\omega_{0} ; \\
\omega_{3}=\frac{\omega_{0}}{\sqrt{1-\sqrt{k_{12}^{2}+k_{23}^{2}}}}
\end{gathered}
$$

Without the second neighbour interaction, one of three splitting frequencies is exactly the same as the resonant frequency $\omega_{0}$. This leads to the belief that power transmission is still maximized at the original resonant frequency as in [7]. This concept may be accurate or inaccurate depending on how the second neighbour mutual coupling takes effect on the system. Therefore, the second neighbour interaction gives a clearer picture of the splitting frequencies in terms of the original resonant frequency and the first and second neighbour coupling coefficients. 


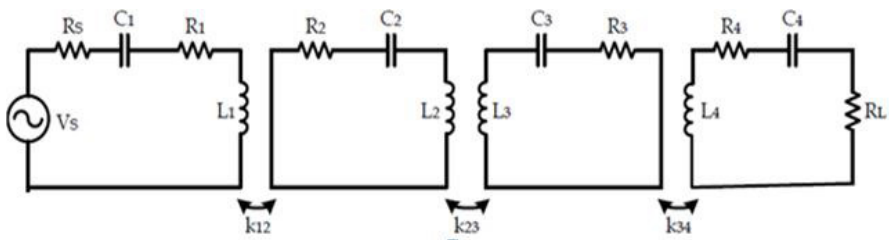

Fig. 1. The four coil system with one transmitter, one receiver and two repeaters.

\section{Four Coil System}

We show how to analyse the system including one transmitter, two repeaters and one receiver affected by the nearest and second neighbour interactions in details by following the same concept:

$$
\begin{gathered}
I_{1}\left(R_{s}+R_{1}+j \omega L_{1}+1 /\left(j \omega C_{1}\right)\right)+ \\
\quad+I_{2}\left(j \omega M_{12}\right)-I_{3}\left(j \omega M_{13}\right)=0
\end{gathered}
$$

By dividing Equation (9) by $j \omega L_{1}$ and assuming that $\left(R_{s}+R_{1}\right) / j \omega L_{1}$ approaches 0 , Equation (9) can be written as:

$$
I_{1}\left(1-\frac{\omega_{0}^{2}}{\omega^{2}}\right)+I_{2} k_{12} \sqrt{\frac{L_{2}}{L_{1}}}-I_{3} k_{13} \sqrt{\frac{L_{3}}{L_{1}}}=0
$$

Similarly, the other three equations are given as:

$$
\begin{gathered}
I_{1} k_{12} \sqrt{\frac{L_{1}}{L_{2}}}+I_{2}\left(1-\frac{\omega_{0}^{2}}{\omega^{2}}\right)-I_{3} k_{23} \sqrt{\frac{L_{3}}{L_{2}}}+ \\
+I_{4} k_{24} \sqrt{\frac{L_{4}}{L_{2}}}=0 \\
-I_{1} k_{13} \sqrt{\frac{L_{1}}{L_{3}}}-I_{2} k_{23} \sqrt{\frac{L_{2}}{L_{3}}}+I_{3}\left(1-\frac{\omega_{0}^{2}}{\omega^{2}}\right)- \\
\quad-I_{4} k_{34} \sqrt{\frac{L_{4}}{L_{3}}}=0 \\
I_{2} k_{24} \sqrt{\frac{L_{2}}{L_{4}}}-I_{3} k_{34} \sqrt{\frac{L_{3}}{L_{4}}}+I_{4}\left(1-\frac{\omega_{0}^{2}}{\omega^{2}}\right)=0
\end{gathered}
$$

We can transform these four simultaneous equations into the matrix form:

$$
\begin{gathered}
{\left[\begin{array}{cccc}
1 & k_{12} \sqrt{\frac{L_{2}}{L_{1}}} & -k_{13} \sqrt{\frac{L_{3}}{L_{1}}} & 0 \\
k_{12} \sqrt{\frac{L_{1}}{L_{2}}} & 1 & -k_{23} \sqrt{\frac{L_{3}}{L_{2}}} & k_{24} \sqrt{\frac{L_{4}}{L_{2}}} \\
-k_{13} \sqrt{\frac{L_{1}}{L_{3}}} & -k_{23} \sqrt{\frac{L_{2}}{L_{3}}} & 1 & -k_{34} \sqrt{\frac{L_{4}}{L_{3}}} \\
0 & k_{24} \sqrt{\frac{L_{2}}{L_{4}}} & -k_{34} \sqrt{\frac{L_{3}}{L_{4}}} & 1
\end{array}\right]\left[\begin{array}{c}
I_{1} \\
I_{2} \\
I_{3} \\
I_{4}
\end{array}\right]=} \\
=\frac{\omega_{0}^{2}}{\omega^{2}}\left[\begin{array}{c}
I_{1} \\
I_{2} \\
I_{3} \\
I_{4}
\end{array}\right]
\end{gathered}
$$

It is observed that matrix A whose diagonal elements are all 1s has symmetry through its diagonal if the self-inductance of coils are identical to each other. In other words, it is clearly seen that $\mathbf{A}(i, j) \times \mathbf{A}(j, i)=k_{i j}^{2}$. In addition, $\mathbf{A}(1,4)$ and $\mathbf{A}(4,1)$ are both equal to zero since we assumed that the third neighbour interaction is not responsible for the mutual coupling of the circuit. Based on three matrices for three different systems above, the rules to obtain the general matrix A with dimensions $n$ by $n$ are expressed in the following way as $\mathbf{A}(i, i)=1$ and $|\mathbf{A}(i, j)|=k_{i j} \sqrt{L_{j} / L_{i}}$ where $k_{i j}=k_{j i}$.

From Equation (14), the Eigen value equation can be obtained as the following:

$$
\begin{gathered}
(1-\lambda)^{4}-(1-\lambda)^{2}\left(k_{12}^{2}+k_{23}^{2}+k_{34}^{2}+k_{13}^{2}+k_{24}^{2}\right)+ \\
+2(1-\lambda)\left(k_{12} k_{13} k_{23}+k_{34} k_{24} k_{23}\right)+k_{13}^{2} k_{24}^{2}+k_{12}^{2} k_{34}^{2} \\
-2 k_{12} k_{13} k_{24} k_{34}=0
\end{gathered}
$$

Through these steps to Equation (15), it is learned that only coupling coefficients have effects on the solutions of Equation (15), which determines the splitting frequencies. By factorizing, Equation (15) becomes:

$$
\begin{gathered}
\left\lfloor(1-\lambda)^{2}-\left(k_{12}^{2}+k_{24}^{2}\right)\right\rfloor\left\lfloor(1-\lambda)^{2}-\left(k_{13}^{2}+k_{34}^{2}\right)\right\rfloor= \\
{\left[k_{23}(1-\lambda)-\left(k_{12} k_{13}+k_{34} k_{24}\right)\right]^{2}}
\end{gathered}
$$

The assumed condition such that $k_{12}^{2}+k_{24}^{2}=k_{13}^{2}+k_{34}^{2}$ is satisfied as long as the radii of all coils are identical and the distance from the transmitting coil to the nearest repeater is the same as the distance from the receiving coil to the nearest repeater. In other words, this assumption means that $k_{12}=k_{34}$ and $k_{13}=k_{24}$ and therefore enables us to simplify Equation (16) further to achieve the result as:

$$
\begin{gathered}
{\left[(1-\lambda)^{2}-\left(k_{12}^{2}+k_{24}^{2}\right)\right]^{2}=\left[k_{23}(1-\lambda)-\left(k_{12} k_{13}\right.\right.} \\
\left.\left.+k_{34} k_{24}\right)\right]^{2}
\end{gathered}
$$

Since $\lambda=\omega_{0}^{2} / \omega^{2}$ or $\omega=\omega_{0} / \sqrt{\lambda}$ the splitting resonant frequencies are:

$$
\begin{aligned}
\omega_{1,2} & =\frac{\omega_{0}}{\sqrt{1+\frac{k_{23} \pm \sqrt{k_{23}^{2}+4\left(k_{12}+k_{13}\right)^{2}}}{2}}} ; \\
\omega_{3,4} & =\frac{\omega_{0}}{\sqrt{1-\frac{k_{23} \pm \sqrt{k_{23}^{2}+4\left(k_{12}+k_{13}\right)^{2}}}{2}}}
\end{aligned}
$$

The result in Equation (18) shows that the splitting resonant frequencies differ from the conventional resonant frequency $\omega_{0}$. Therefore the relationship between splitting frequency and coupling coefficients with regards to the nearest and second neighbour interactions is demonstrated clearly. In addition, because of the conditions: $k_{12}=k_{34}$ and $k_{13}=k_{24}$, there always exist four splitting frequencies that are either complex or real. In the case that the second neighbour interaction is not included $\left(k_{13}=k_{24}=0\right)$, the resultant frequencies in Equation (18) are the same as in [7]. Obviously, the result could be more accurate if the second neighbour coupling which also accounts for this inductive circuit behaviour is considered. In summary, the result for the four coil system in this paper is more realistic compared to result in [7] because it shows how the second neighbour interaction affects the splitting frequencies in addition to the nearest one. 


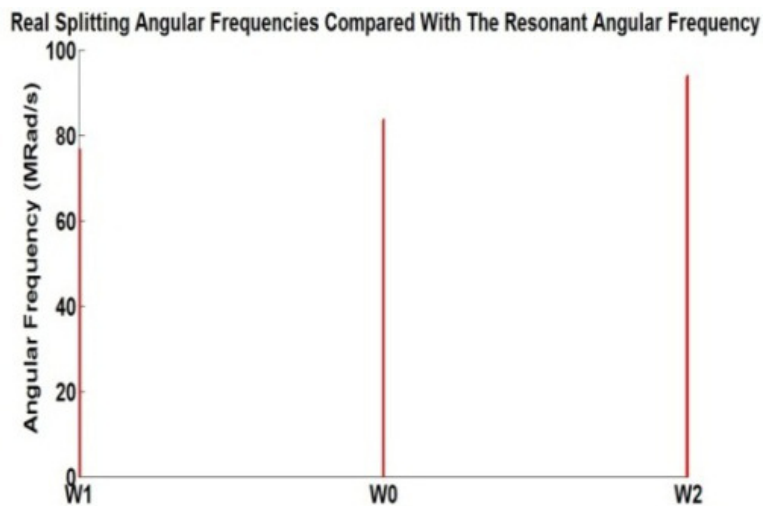

Fig. 2. Two frequencies for the two coil system compared with the resonant frequency.
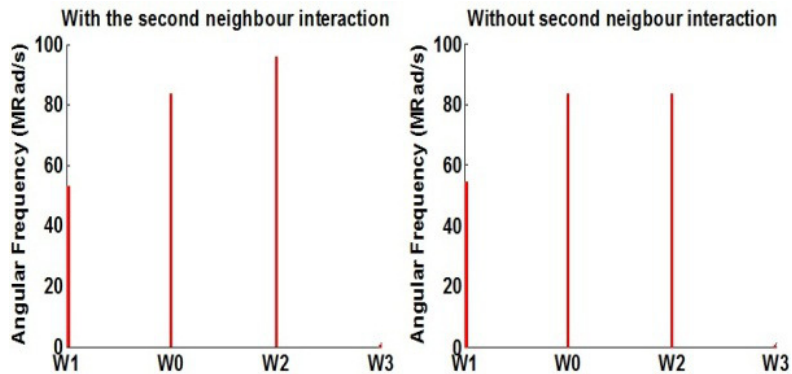

Fig. 3. Two real splitting frequencies for the three coil system compared with the resonant frequency.

Although the assumed condition above leads to four splitting frequencies, this limitation is not true all the time so that numerical methods could be used to get the approximate results for Equation (15).

\section{Simulations FOR the Splitting AND Resonant FREQUENCIES}

The simulations in this section are based on the following conditions: Self- inductance of the coil is: $L=0.5 \mu \mathrm{H}$; Capacitance is: $C=286 \mathrm{pF}$ and the traditional resonant angular frequency is: $\omega_{0}=84 \mathrm{MRad} / \mathrm{s}$.

\section{A. Two Coil System}

The Eigen value Equation (3) is run by MATLAB. For any value of $k_{12}$, two modes have been achieved.

The result shown in Fig. 2 where $k_{12}=0.2$ demonstrates two splitting frequencies standing for two modes. This achievement has also been proved in Equation (4).

\section{B. Three Coil System}

The Eigen value Equation (6) is used for MATLAB simulation so that a clear picture of splitting frequencies with the effect of second neighbour interaction can be demonstrated.

Two modes have been observed as both $k_{23}$ and $k_{12}$ are set to 0.95 and $k_{13}$ is set to 0.2375 . It is seen that putting coils close to each other brings one negative Eigen solution for Equation (6), which eventually leads to one complex
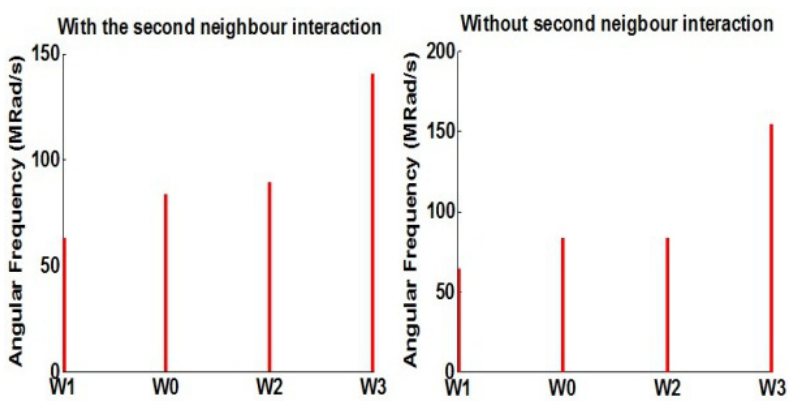

Fig. 4. Three splitting frequencies for the three coil system compared with the resonant frequency.

frequency. This result can be seen obviously in Fig. 3 where $\omega_{3}$ is set to zero due to complex frequency omission. Besides, the right side of Fig. 3 shows that one of the splitting frequencies is the same as $\omega_{0}$, which is proved in Equation (8). This result will be true for any three coil system without the second neighbour interaction.

Three modes have been gained for most of the values of the coupling coefficients. For example, when $k_{12}=k_{23}=0.5$ and $k_{13}=0.125$ in Fig. 4, three different splitting frequencies or three different modes are observed. Once again, the splitting is affected by the second neighbour interaction showing a difference from the system without this coupling as in Fig. 4. All the three frequencies in the left side are not the same as $\omega_{0}$ and they also differ from those in the right one. The same consequence is observed with $k_{23}=0.7 ; k_{12}=0.8$; $k_{13}=0.175$ as shown clearly in Fig. 5 .

\section{Four Coil System}

Equation (15) is used for simulation in MATLAB to get the accurate results without any constraint to the four coil systems.

Two modes have been obtained in Fig. 6 for the four coil system when $k_{12}=0.1376, k_{23}=0.01, k_{34}=0.1343$, $k_{13}=0.0025, k_{24}=0.0025$. This result is the same as in [2] in the way that there are two pairs of splitting frequencies standing for two splitting modes since the same values for the nearest neighbour coupling coefficients are used. It is seen in Fig. 6 that the four splitting frequencies do not coincide with the traditional resonant frequency and only two separate pairs of frequencies can be achieved from the four ones. The reason is that Equation 15 gives two pairs of values that are different with unity. It can be learned that coupling coefficients will decide the coefficients for the quartic Equation (15), which leads to the Eigen roots and eventually to the splitting frequencies.

Similarly, frequency splitting for three modes have been investigated when $k_{12}=0.1, k_{23}=0.6, k_{34}=0.05$, $k_{13}=0.025, k_{24}=0.0125$. This result is quite different from the result in [1]. When $k_{12}$ and $k_{34}$ are the same values, as long as $k_{23}$ in paper [1] is greater 0 , the system in [1] has three modes if it includes the traditional resonant mode. However, in this work, only when $k_{23}$ starts to be greater than 0.3 , three modes (one mode is the same as the traditional resonant one) can be observed as $k_{23}=0.6$ in Fig. 7. Otherwise, our four coil system will have four modes. 

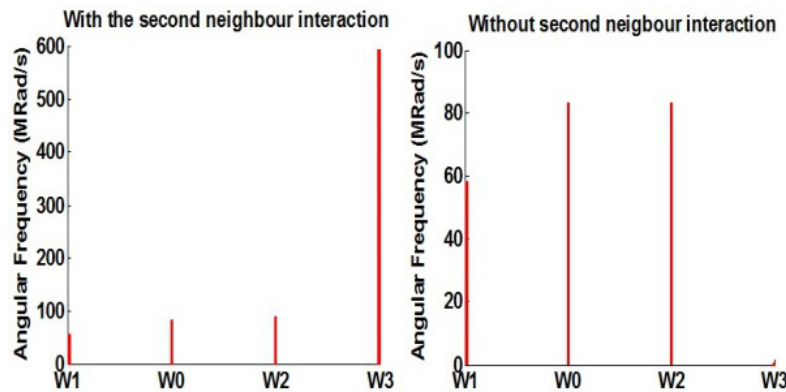

Fig. 5. Three/two real splitting frequencies for the three coil system compared with the resonant frequency.
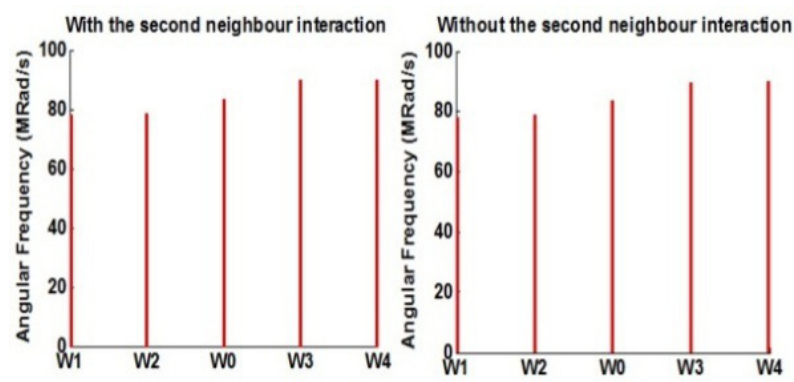

Fig. 6. Two splitting frequencies for the four coil system compared with resonant frequency.

Another case for three modes was observed as $k_{12}=0.9$; $k_{23}=0.5 ; k_{34}=0.7 ; k_{13}=0.125 ; k_{24}=0.125$. This time, Equation (15) brings three real and one complex Eigen value. The complex value is omitted since we only deal with real frequencies. This is why the three separate splitting frequencies are different from $\omega_{0}$ and one frequency is set to 0 . We notice that the frequency values are not the same for the system with second neighbour coupling coefficients in the left of Fig. 8 and without it in the right of Fig. 8. This result is not found in Fig. 6 and Fig. 7. Therefore, the second neighbour coupling does have significant effect on the system behaviour depending on its degree. In other words, if $k_{13}$ and $k_{24}$ are big enough, they will affect the circuit behaviour. Thus, the second neighbour interaction should be included in the system considerations if coils are arranged closely to each other.

Finally, four modes have been obtained as well when $k_{12}=$ $0.3 ; k_{23}=0.6 ; k_{34}=0.8 ; k_{13}=0.075 ; k_{24}=0.15$. In this case, four real splitting are frequencies observed for the system where the second neighbour coupling is involved as seen in the left of Fig. 9. Nevertheless, only three real splitting frequencies could be achieved for this system without $k_{13}$ and $k_{24}$ as in the right of Fig. 9. Thus, the effect of second neighbour interaction is shown again in this case.

To sum up, the differences of simulation results between systems with and without the second neighbour interaction have been shown more clearly in Tab. I located at the last page. The results are the same only for the four coil system in the case of two modes with and without the second neighbour interaction. For other cases, the splitting frequencies for systems with the second interaction are quite different from the ones without it.
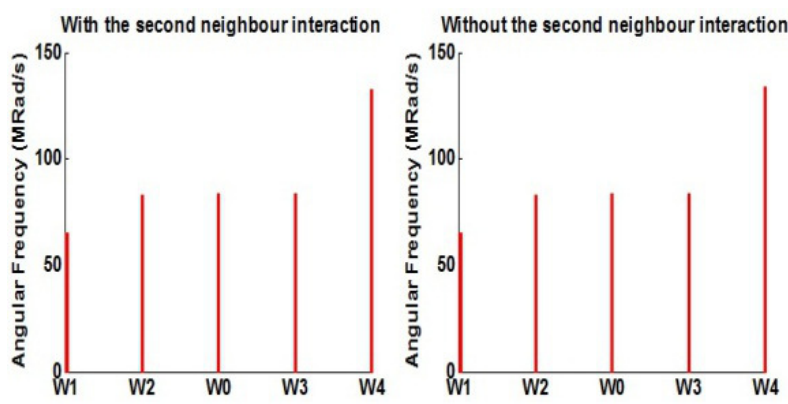

Fig. 7. Three splitting frequencies for the four coil system compared with the resonant frequency.
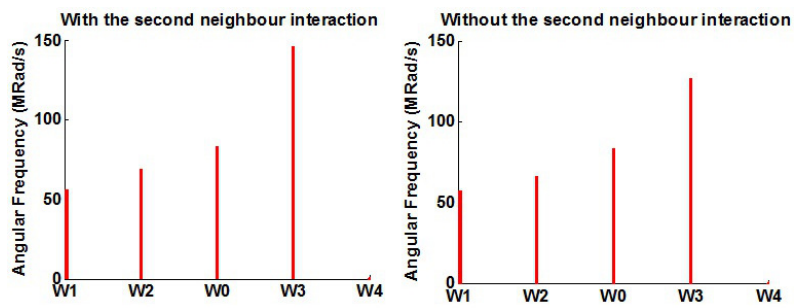

Fig. 8. Three real splitting frequencies for the four coil system compared with the resonant frequency.

\section{TRANSFER FunCtions FOR INDUCTIVE Systems}

In order to estimate the power efficiency of each inductive system at different frequencies, the transfer functions of the models are derived in terms of angular frequencies. Besides, they are also used for assessing the system gains in terms of quality factors and coupling coefficients at resonance as to either find out the critical coupling coefficients at the resonant frequencies or to simplify the models further in some specific conditions.

\section{A. Two Coil System}

The circuit can be summarised in the following matrix form:

$$
\left[\begin{array}{cc}
Z_{1} & j \omega M_{12} \\
j \omega M_{12} & Z_{2}
\end{array}\right]\left[\begin{array}{c}
I_{1} \\
I_{2}
\end{array}\right]=\left[\begin{array}{c}
V_{s} \\
0
\end{array}\right]
$$

The current flowing in the receiver side is:

$$
I_{2}=\frac{-j \omega M_{12} V_{S}}{Z_{1} Z_{2}+\omega^{2} M_{12}^{2}}
$$

The voltage at the receiver load is:

$$
V_{L}=-I_{2} R_{L}
$$

Therefore, the voltage gain is derived as:

$$
\frac{V_{L}}{V_{S}}=\frac{j \omega M_{12} R_{L}}{Z_{1} Z_{2}+\omega^{2} M_{12}^{2}}
$$

Voltage gain can be expressed in another way in terms of quality factors and coupling coefficients. Consider the quality factors of the transmitting coil and receiving coil as the following below:

$$
Q_{1}=\frac{\omega L_{1}}{R_{1}+R_{S}}, \quad Q_{2}=\frac{\omega L_{2}}{R_{2}+R_{L}}
$$




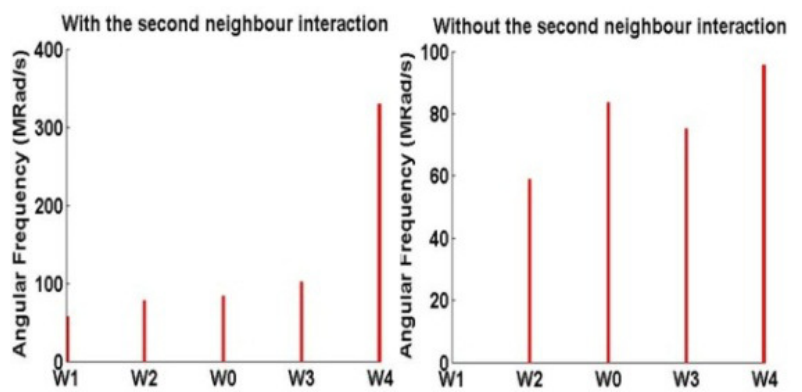

Fig. 9. Four/three real splitting frequencies for the four coil system compared with the resonant frequency.

At resonance, all the impedances become pure resistances. Thus, Equation (22) can be written as the follows:

$$
\begin{gathered}
\frac{V_{L}}{V_{S}}=\frac{j k_{12} \sqrt{Q_{1} Q_{2}} \sqrt{\left(R_{1}+R_{S}\right)\left(R_{2}+R_{L}\right)} R_{L}}{\left(R_{1}+R_{S}\right)\left(R_{2}+R_{L}\right)} \\
+k_{12}^{2} Q_{1} Q_{2}\left(R_{1}+R_{S}\right)\left(R_{2}+R_{L}\right)
\end{gathered}
$$

By simplifying Equation (24) and taking the absolute value of the voltage gain, we can obtain this expression:

$$
\left|\frac{V_{L}}{V_{S}}\right|=\frac{R_{L}}{\sqrt{\left(R_{1}+R_{S}\right)\left(R_{2}+R_{L}\right)}} \frac{k_{12} \sqrt{Q_{1} Q_{2}}}{1+k_{12}^{2} Q_{1} Q_{2}}
$$

This result here is the same as paper [8]. The only minor difference is that the source resistance and the load resistance may be ignored for simplification [8].

Equation (25) helps to determine the critical coupling coefficient at resonance that enables the voltage gain to achieve the maximum value. Thus, by differentiating Equation (25) in terms of $k_{12}$ and letting the first derivative of that equation equal to 0 , the following equation can be gained:

$$
\frac{\sqrt{Q_{1} Q_{2}}\left(1+k_{12}^{2} Q_{1} Q_{2}-2 k_{12}^{2} Q_{1} Q_{2}\right.}{\left(1+k_{12}^{2} Q_{1} Q_{2}\right)^{2}}=0
$$

The solution for Equation (26) brings the critical coupling value which is:

$$
k_{12}=\frac{1}{\sqrt{Q_{1} Q_{2}}}
$$

Moreover, in both loosely coupled and low quality factor conditions [8], Equation (25) can be shortened into:

$$
\left|\frac{V_{L}}{V_{S}}\right|=\frac{R_{L} k_{12} \sqrt{Q_{1} Q_{2}}}{\sqrt{\left(R_{1}+R_{S}\right)\left(R_{2}+R_{L}\right)}}
$$

and

$$
\left|\frac{V_{L}}{V_{S}}\right|=\beta_{2}\left(k_{12} \sqrt{Q_{1} Q_{2}}\right)
$$

where $\beta_{2}=R_{L} / \sqrt{\left(R_{1}+R_{S}\right)\left(R_{2}+R_{L}\right)}$

It is said that at resonance, with weak coupling and low quality factor cases, the transfer function for inductive twocoil system is therefore linearly proportional to the coupling coefficient, which does not apply for other cases.

\section{B. Three Coil System}

For three coil system, the matrix form representing the system performance can be expressed as given below:

$$
\left[\begin{array}{ccc}
Z_{1} & j \omega M_{12} & -j \omega M_{13} \\
j \omega M_{12} & Z_{2} & -j \omega M_{23} \\
-j \omega M_{13} & -j \omega M_{23} & Z_{3}
\end{array}\right]\left[\begin{array}{c}
I_{1} \\
I_{2} \\
I_{3}
\end{array}\right]=\left[\begin{array}{c}
V_{S} \\
0 \\
0
\end{array}\right]
$$

The current flowing in the receiver loop is derived in Equation (30) below.

The load voltage at the receiver side is:

$$
V_{L}=I_{3} R_{L}
$$

Thus, the voltage gain for this three coil system is given as Equation (32) below.

In the same way, the voltage gain for three coil system can also be represented in terms of quality factors and coupling coefficients. Let the quality factors for transmitting, intermediate and receiving coils be:

$$
Q_{1}=\frac{\omega L_{1}}{R_{1}+R_{S}}, \quad Q_{2}=\frac{\omega L_{2}}{R_{2}}, \quad Q_{3}=\frac{\omega L_{3}}{R_{3}+R_{L}}
$$

By substituting the quality factors into Equation (32), the inductive transfer function for three coil system at resonance can be obtained as the expression:

$$
\frac{V_{L}}{V_{S}}=\frac{R_{L}}{\sqrt{\left(R_{1}+R_{S}\right)\left(R_{3}+R_{L}\right)}}
$$

$$
\cdot \frac{\left(k_{12} k_{23} Q_{2}+j k_{13}\right) \sqrt{Q_{1} Q_{3}}}{k_{12}^{2} Q_{1} Q_{2}+k_{23}^{2} Q_{2} Q_{3}+k_{13}^{2} Q_{1} Q_{3}+1-2 j k_{12} k_{23} k_{13} Q_{1} Q_{2} Q_{3}}
$$

The result in Equation (34) differs from the transfer function for three coil system in paper [8] due to the existence of the second neighbour mutual interaction. Based on Equation (34), the critical coupling coefficients for three coil systems at the resonant frequency are the solutions for three simultaneous equations which are the partial derivatives of Equation (34). The general solutions for these equations are left for mathematicians.

In addition, in the case of both loosely coupled (the first neighbour coupling coefficients are quite small and thus the second neighbour coupling coefficient does not account for system operation) and low quality factors $Q$, the voltage gain for the system can reduce to:

$$
\left|\frac{V_{L}}{V_{S}}\right|=\frac{R_{L} k_{12} k_{23} Q_{2} \sqrt{Q_{1} Q_{3}}}{\sqrt{\left(R_{1}+R_{S}\right)\left(R_{3}+R_{L}\right)}}
$$

and

$$
\left|\frac{V_{L}}{V_{S}}\right|=\beta_{3}\left(k_{12} \sqrt{Q_{1} Q_{2}}\right)\left(k_{23} \sqrt{Q_{2} Q_{3}}\right)
$$

where $\beta_{3}=R_{L} / \sqrt{\left(R_{1}+R_{S}\right)\left(R_{3}+R_{L}\right)}$

Equation (35) is exactly the same result in paper [8] due to omission of the second neighbour coupling coefficient. This equation is used only when coils are arranged quite far from each other and have low quality factors. Otherwise, Equation (34) definitely has to be used for other scenarios. 


$$
\begin{aligned}
I_{3} & =\frac{\left(\omega^{2} M_{12} M_{23}+j \omega M_{13} Z_{2}\right) V_{S}}{\omega^{2}\left(Z_{1} M_{23}^{2}+Z_{2} M_{13}^{2}+Z_{3} M_{12}^{2}\right)-2 j \omega^{3} M_{12} M_{23} M_{13}+Z_{1} Z_{2} Z_{3}} \\
\frac{V_{L}}{V_{S}} & =\frac{\left(\omega^{2} M_{12} M_{23}+j \omega M_{13} Z_{2}\right) R_{L}}{\omega^{2}\left(Z_{1} M_{23}^{2}+Z_{2} M_{13}^{2}+Z_{3} M_{12}^{2}\right)-2 j \omega^{3} M_{12} M_{23} M_{13}+Z_{1} Z_{2} Z_{3}}
\end{aligned}
$$

\section{Four Coil System}

The four coil system can be transformed into the characteristic matrix as the following:

$$
\left[\begin{array}{cccc}
Z_{1} & j \omega M_{12} & -j \omega M_{13} & 0 \\
j \omega M_{12} & Z_{2} & -j \omega M_{23} & j \omega M_{24} \\
-j \omega M_{13} & -j \omega M_{23} & Z_{3} & -j \omega M_{34} \\
0 & j \omega M_{24} & -j \omega M_{34} & Z_{4}
\end{array}\right]\left[\begin{array}{c}
I_{1} \\
I_{2} \\
I_{3} \\
I_{4}
\end{array}\right]=\left[\begin{array}{c}
V_{S} \\
0 \\
0 \\
0
\end{array}\right]
$$

The current flowing in the receiver loop is given in Equation (37) put on the top of next page.

The load voltage at the receiver side is:

$$
V_{L}=-L_{4} R_{L}
$$

The voltage gain is finally obtained as the following Equation (39) put on the top of next page.

The voltage gain in Equation (39) can be written in terms of the quality factors and coupling coefficients in the same way with two and three coil systems. Quality factors for four coil system are defined as the following:

$$
\begin{aligned}
Q_{1} & =\frac{\omega L_{1}}{R_{1}+R_{S}} ; \quad Q_{2}=\frac{\omega L_{2}}{R_{2}} ; \\
Q_{3} & =\frac{\omega L_{3}}{R_{3}} ; \quad Q_{4}=\frac{\omega L_{4}}{R_{4}+R_{L}}
\end{aligned}
$$

At resonance, Equation (39) can turn into the following inductive transfer function as given in Equation (41) put on the top of next page.

Comparing the transfer function Equation (41) with the inductive transfer function in paper [8], it is noticed that there is a remarkable difference because of considering the second neighbour interaction in the four coil model.

It is learnt that the critical values are the roots for four simultaneous equations that are the first partial derivatives of Equation (41). Solving those equations in order to have the general solutions involves more analysis.

Besides, in the low coupling and small quality factor scenario, Equation (41) can be simplified further into the following expression:

$$
\left|\frac{V_{L}}{V_{S}}\right|=\frac{R_{L} k_{12} k_{23} k_{34} Q_{2} Q_{3} \sqrt{Q_{1} Q_{4}}}{\sqrt{\left(R_{1}+R_{S}\right)\left(R_{4}+R_{L}\right)}}
$$

and

$$
\left|\frac{V_{L}}{V_{S}}\right|=\beta_{4}\left(k_{12} \sqrt{Q_{1} Q_{2}}\right)\left(k_{23} \sqrt{Q_{2} Q_{3}}\right)\left(k_{34} \sqrt{Q_{3} Q_{4}}\right)
$$

where $\beta_{4}=R_{L} / \sqrt{\left(R_{1}+R_{S}\right)\left(R_{4}+R_{L}\right)}$.

The result obtained in Equation (42) is similar to the one in paper [8] and only useful for special conditions as mentioned

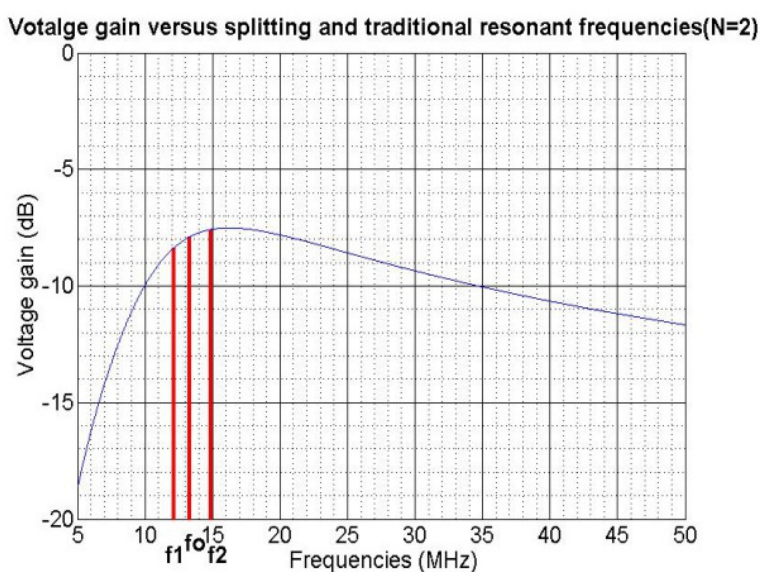

Fig. 10. Voltage gain versus splitting frequencies for two-coil system in Equation (22)

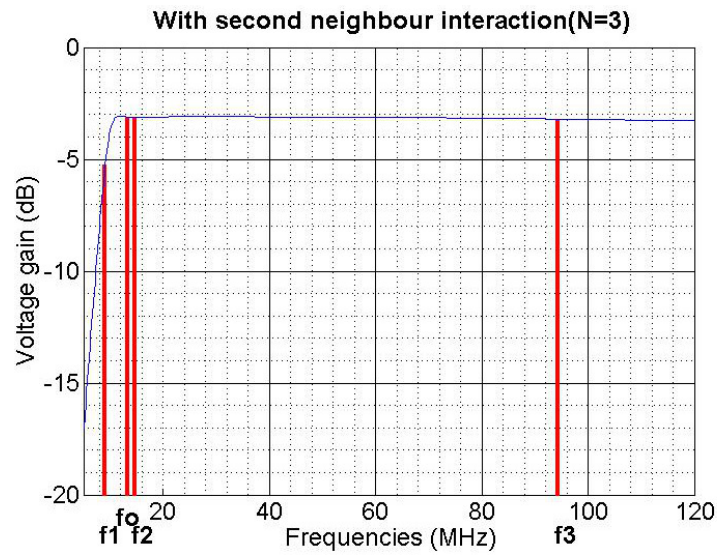

Fig. 11. Voltage gain versus splitting frequencies for three-coil system in Equation (32) with three modes.

above. Therefore, for general cases, Equation (41) has to be deployed instead.

In general, the inductive transfer function for $N$ coil system can be written as the product:

$$
\left|\frac{V_{L}}{V_{S}}\right|=\beta_{N} \prod_{j=1}^{N}\left(k_{j, j+1} \sqrt{Q_{j} Q_{j+1}}\right.
$$

where $\beta_{N}=R_{L} / \sqrt{\left(R_{1}+R_{S}\right)\left(R_{N}+R_{L}\right)}$

Equation (43) is derived through the rules acknowledged from transfer functions for two, three and four coil systems. This equation shows the relationship of the voltage gain in terms of coupling coefficients, quality factors and resistances in any inductive system at loosely coupling and low quality factor conditions. 


$$
\begin{aligned}
& I_{4}=\frac{V_{S}\left[-\omega^{2}\left(M_{24} M_{12} Z_{3}+M_{13} M_{34} Z_{2}\right)+j \omega^{3}\left(M_{13} M_{23} M_{24}+M_{34} M_{12} M_{23}\right)\right]}{\omega^{4}\left(M_{12}^{2} M_{34}^{2}-2 M_{12} M_{34} M_{13} M_{24}+M_{13}^{2} M_{24}^{2}\right)-2 j \omega^{3}\left(M_{23} M_{24} M_{34} Z_{1}+M_{23} M_{12} M_{13} Z_{4}\right)} \\
& +\omega^{2}\left(M_{12}^{2} Z_{3} Z_{4}+M_{23}^{2} Z_{1} Z_{4}+M_{34}^{2} Z_{1} Z_{2}+M_{13}^{2} Z_{2} Z_{4}+M_{24}^{2} Z_{1} Z_{3}\right)+Z_{1} Z_{2} Z_{3} Z_{4} \\
& \frac{V_{L}}{V_{S}}=\frac{R_{L}\left[-\omega^{2}\left(M_{24} M_{12} Z_{3}+M_{13} M_{34} Z_{2}\right)+j \omega^{3}\left(M_{13} M_{23} M_{24}+M_{34} M_{12} M_{23}\right)\right]}{\omega^{4}\left(M_{12}^{2} M_{34}^{2}-2 M_{12} M_{34} M_{13} M_{24}+M_{13}^{2} M_{24}^{2}\right)-2 j \omega^{3}\left(M_{23} M_{24} M_{34} Z_{1}+M_{23} M_{12} M_{13} Z_{4}\right)} \\
& +\omega^{2}\left(M_{12}^{2} Z_{3} Z_{4}+M_{23}^{2} Z_{1} Z_{4}+M_{34}^{2} Z_{1} Z_{2}+M_{13}^{2} Z_{2} Z_{4}+M_{24}^{2} Z_{1} Z_{3}\right)+Z_{1} Z_{2} Z_{3} Z_{4} \\
& \frac{V_{L}}{V_{S}}=\frac{R_{L}}{\sqrt{\left(R_{1}+R_{S}\right)\left(R_{4}+R_{L}\right)}} \frac{\sqrt{Q_{1} Q_{4}}\left(-k_{12} k_{24} Q_{2}-k_{13} k_{34} Q_{3}+j Q_{2} Q_{3} k_{23}\left(k_{13} k_{24}+k_{12} k_{34}\right)\right)}{Q_{1} Q_{2} Q_{3} Q_{4}\left(k_{12} k_{34}-k_{13} k_{24}\right)^{2}-2 j Q_{2} Q_{3} k_{23}\left(k_{12} k_{13} Q_{1}+k_{24} k_{34} Q_{4}\right)} \\
& +k_{12}^{2} Q_{1} Q_{2}+k_{23}^{2} Q_{2} Q_{3}+k_{13}^{2} Q_{1} Q_{3}+k_{24}^{2} Q_{2} Q_{4}+k_{34}^{2} Q_{3} Q_{4}+1
\end{aligned}
$$

\section{Simulations for Voltage Gains at Different FREQUENCIES}

The simulations in section IV are based on the same conditions with section II. Voltage gains for all systems have been plotted versus the splitting and traditional resonant frequencies in order to compare the differences of power efficiency at different frequencies and then determine the maximum efficiency of systems when mode splitting happens.

It is noticed that for two coil system with the same coupling coefficient with previous section $k_{12}=0.2$, there are two splitting frequencies which are different with the resonant frequency as proved in Equation (4) and section II. From Fig. 10, it is learnt that the maximum voltage gain can be achieved at the splitting frequency $f_{2}$ instead of the resonant frequency $f_{0}$. It is said that in the strongly coupled case, frequency splitting degrades the system performance in term of power transfer. Therefore, correct frequency selection is supposed to perform to achieve the maximum power efficiency.

For the three coil systems, we have done the simulation with the similar conditions as one case in section II where $k_{23}=0.7 ; k_{12}=0.8 ; k_{13}=0.175$. The results obtained in Figs. 11 and 12 agree with all the ones achieved in section II for systems with and without the second neighbour interaction. From these two figures, it is learnt that the system performance is not affected by the mode splitting phenomenon.

Similarly, we have achieved the same results as the previous part in section III for the four coil systems where $k_{12}=0.3$; $k_{23}=0.6 ; k_{34}=0.8 ; k_{13}=0.075 ; k_{24}=0.15$. Nevertheless, in this situation, the maximum voltage gain cannot be gained at the traditional resonant frequency as demonstrated in Figs. 13 and 14, which again strengthens the statement that for strongly coupling scenarios, mode splitting does affect the system performances due to power transfer efficiency. Thus, it is supposed to choose another splitting frequency instead of the resonant one in order to achieve the maximum power transfer.

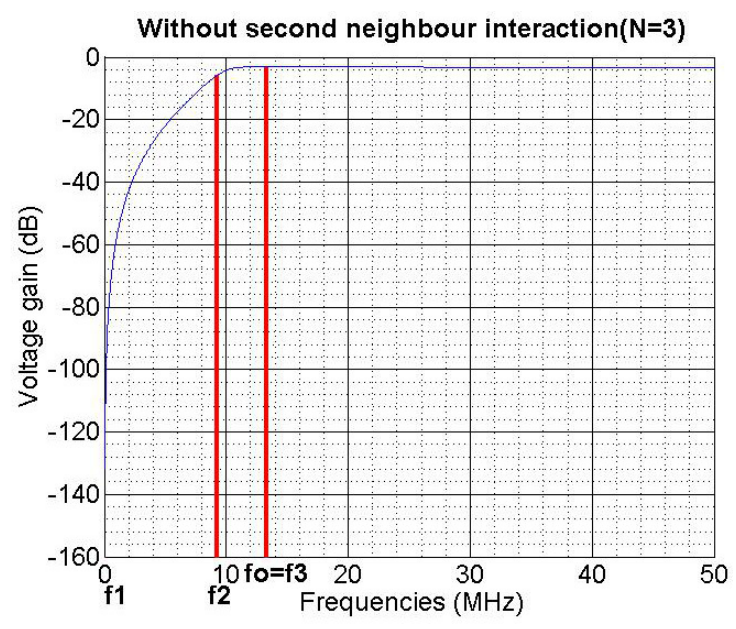

Fig. 12. Voltage gain versus splitting frequencies for three-coil system in Equation (32) with two modes.

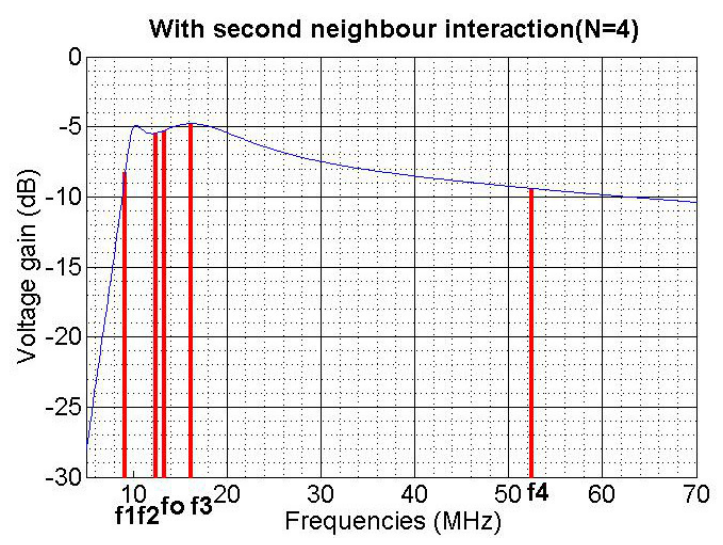

Fig. 13. Voltage gain versus splitting frequencies for four-coil system in Equation (39) with 4modes.

\section{CONCLUSION}

From the circuit analysis and simulation result parts, it is believed that the only factor affecting the mode splitting phenomenon is the coupling coefficients. The degree of these 


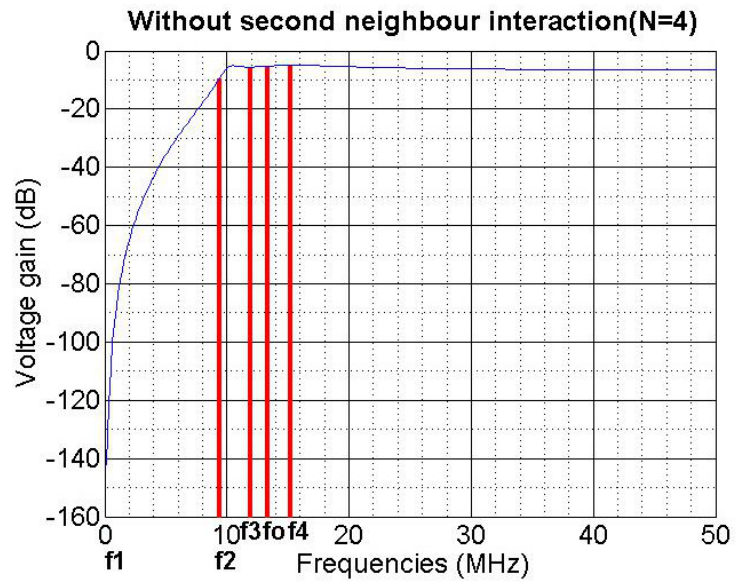

Fig. 14. Voltage gain versus splitting frequencies for the four coil system in Equation (39) with 3 modes.

TABLE I

COMPARISON OF Simulation RESUlTS With/Without SECOND NEIGHBOUR INTERACTION

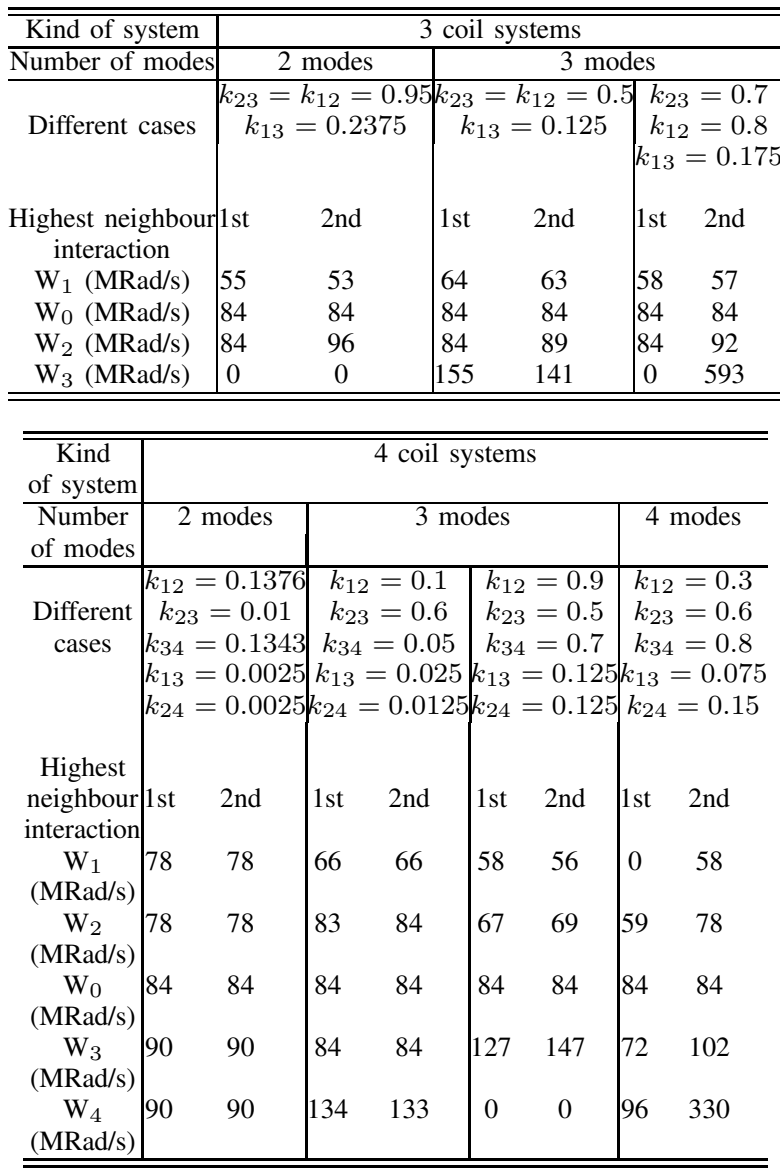

coupling coefficients plays a very important role in producing the Eigen values that result in the number of real splitting frequencies. The conventional resonant frequency does not decide the number of modes but alter the magnitudes of the splitting frequencies due to its relationship with Eigen values and these frequencies. Moreover, the second neighbour interaction has strong effects on the system behaviour regarding to splitting frequency results, especially when the distances between coils are short as in applications such as wireless charging platforms and inductive power chains. Thus, this factor needs to be considered in any system analysis. In wireless power transfer, mode splitting should be avoided because of power transmission efficiency. It has been observed that the application of resonance with loops limits inductive transceivers to monochromatic applications that use only the conventional resonant frequency as the operating frequency of the system. Nevertheless, splitting frequency phenomenon can be utilized for data transmission at many separate frequencies concurrently. In this work, further insights into mode splitting have enabled to analyse the power efficiency for inductive systems at splitting and resonant frequencies so that the correct frequency can be tuned. Besides, coupling coefficients can be determined at resonance by representing the transfer functions in another way. Also the simplified forms of voltage gains have brought the general system model for $N$-coil near field inductive system.

\section{APPENDIX}

\section{DEFINITIONS FOR ALL VARIABLES}

\begin{tabular}{|c|c|}
\hline Variables & Definitions \\
\hline$k_{12}, k_{21}$ & Coupling coefficient between coils 1 and 2 \\
\hline$k_{23}, k_{32}$ & Coupling coefficient between coils 2 and 3 \\
\hline$k_{34}, k_{43}$ & Coupling coefficient between coils 3 and 4 \\
\hline$k_{13}, k_{31}$ & Coupling coefficient between coils 1 and 3 \\
\hline$k_{24}, k_{42}$ & Coupling coefficient between coils 2 and 4 \\
\hline$M_{12}, M_{21}$ & Mutual Inductance between coils 1 and 2 \\
\hline$M_{23}, M_{32}$ & Mutual Inductance between coils 2 and 3 \\
\hline$M_{34}, M_{43}$ & Mutual Inductance between coils 3 and 4 \\
\hline$M_{13}, M_{31}$ & Mutual Inductance between coils 1 and 3 \\
\hline$M_{24}, M_{42}$ & Mutual Inductance between coils 2 and 4 \\
\hline $\begin{array}{c}\omega \\
\omega_{0}\end{array}$ & $\begin{array}{l}\text { Angular frequency } \\
\text { Resonant angular frequency }\end{array}$ \\
\hline$f_{0}$ & Resonant frequency \\
\hline$\omega_{1}, \omega_{2}, \omega_{3}, \omega_{4}$ & Splitting angular frequencies \\
\hline$f_{1}, f_{2}, f_{3}, f_{4}$ & Splitting frequencies \\
\hline$Q_{1}, Q_{2}, Q_{3}, Q_{4}$ & Quality factors for coil $1,2,3,4$ \\
\hline$I_{1}, I_{2}, I_{3}, I_{4}$ & Currents flowing in coils $1,2,3,4$ \\
\hline$L_{1}, L_{2}, L_{3}, L_{4}$ & Inductances of coils $1,2,3,4$ \\
\hline$C_{1}, C_{2}, C_{3}, C_{4}$ & Capacitors in series with coils $1,2,3,4$ \\
\hline$R_{1}, R_{2}, R_{3}, R_{4}$ & Resistances of coils $1,2,3,4$ \\
\hline$Z_{1}, Z_{2}, Z_{3}, Z_{4}$ & Impedances for coil $1,2,3,4$ \\
\hline$R_{S}, R_{L}$ & Resistances for source, load \\
\hline$V_{S}, V_{L}$ & Voltages across the source, load \\
\hline
\end{tabular}

\section{REFERENCES}

[1] H. Hoang and F. Bien, "Maximizing Efficiency of Electromagnetic Resonance Wireless Power Transmission Systems with Adaptive Circuits," in Wireless Power Transfer - Principles and Engineering Explorations, K. Y. Kim, Ed., 2012, ISBN: 978-953-307-874-8, InTech.

[2] A. P. Sample, D. A. Meyer, and J. R. Smith, "Analysis, Experimental Results, and Range Adaption of Magnetically Coupled Resonators for Wireless Power Transfer," IEEE Transaction on Industrial Electronics, vol. 58, no. 2, pp. 544-554, February 2011.

[3] B. L. Cannon, J. F. Hoburg, D. D. Stancil, and S. C. Goldstein "Magnetic Resonant Cupling As a Potential Means for Wireless Power Transfer to Multiple Receivers," IEEE Transactions on Power Electronics, vol. 24 no. 7, pp. 1819-1825, July 2009.

[4] J. W. Kim, H.-C. Son, K.-H. Kim, and Y.-J. Park, "Efficiency Analysis of Magnetic Resonance Wireless Power Transfer With Intermediate Resonant Coil," IEEE Antennas and Wireless Propagation Letters, vol. 10 pp. 389-392, 2011. 
[5] T. Imura, H. Okabe, and Y. Hori, "Basic experimental study on helical antennas of wireless power transfer for Electric Vehicles by using magnetic resonant couplings," Proceedings of IEEE Vehicle Power and Propulsion Conference, pp. 936-940, 2009.

[6] A. Scher, "Tutorial: Positive Coupling, Negative Coupling, and All That," If Microwaves Could Talk, 2010.
[7] D. Ahn and S. Hong, "A study on Magnetic Field Repeaters in Wireless Power Transfer," IEEE Industrial Electronics, 14 February 2011, Accepted for publication on 30 January, 2012.

[8] J. I. Agbinya, "A Magneto-Inductive Link Budget for Wireless Power Transfer and Inductive Communication Systems," Progress in Electromagnetics Research C, vol. 37, pp. 15-28, 2013. 